\title{
Neurological adverse events temporally associated to mass vaccination against yellow fever in Juiz de Fora, Brazil, 1999-2005
}

\author{
Guilherme Côrtes Fernandes ${ }^{\mathrm{a}, \mathrm{b}, *}$, Luiz Antonio Bastos Camacho ${ }^{\mathrm{b}}$, Marilia Sá Carvalho ${ }^{\mathrm{b}}$, \\ Maristela Batista ${ }^{c}$, Sonia Maria Rodrigues de Almeida ${ }^{c}$ \\ a Santa Casa de Misericórdia de Juiz de Fora, Minas Gerais, Brazil \\ ${ }^{\mathrm{b}}$ Escola Nacional de Saúde Pública, Fundação Oswaldo Cruz, Rio de Janeiro, Brazil \\ c Departamento de Vigilância Epidemiológica, Prefeitura Municipal de Juiz de Fora, Minas Gerais, Brazil
}

Available online 30 January 2007

\begin{abstract}
The identification of adverse events following immunization (AEFI) and their prompt investigation are important to allow a timely and scientifically based response to the users of immunization services. This article presents an analysis of notified AEFI cases between 1999 and 2005 and their temporal association with 2001 yellow fever vaccination campaign, AEFI notification attributed to yellow fever vaccination rose from 0.06 to 1.32 per 100,000 vaccinees in Brazil, between 1998 and 2000. During the 2001 yellow fever mass vaccination campaign held in Juiz de Fora, Brazil, 12 cases of aseptic meningitis were temporally associated to yellow fever vaccination, but clinical and laboratory data were not available to confirm nor deny causality. Epidemiological studies associated to enhanced surveillance and standardized protocols should take advantage of public health interventions like mass vaccination campaigns and implementation of new vaccination strategies in order to assess and investigate vaccine safety.
\end{abstract}

(C) 2007 Published by Elsevier Ltd.

Keywords: Yellow fever vaccine; Adverse effects; Adverse events following immunization; Brazil; Mass vaccination campaign

\section{Introduction}

The perception of risks and benefits involving the use of vaccines does not always follow scientific criteria [1]. Causality has often been inferred from the mere temporal sequence of facts [2]. The greatest limitation of data from surveillance of adverse events following immunization (AEFI) results from the very definition of AEFI cases: signs or symptoms that follow the application of a vaccine and that are believed to be caused by the vaccine. The great challenge to surveillance of AEFI is to distinguish abnormalities caused by vaccines from those associated to unrelated conditions. The investigation must be swift to preserve the public's confidence

\footnotetext{
* Corresponding author at: Escola Nacional de Saúde Pública, Fundação Oswaldo Cruz, Rua Leopoldo Bulhões, 1480-Sala 820, Manguinhos, 21041-210 Rio de Janeiro, Brazil. Tel.: +55 323234 7387; fax: +552125982630 .

E-mail addresses: gcortes@ensp.fiocruz.br, gcortes@ riscobiologico.org (G.C. Fernandes).
}

and avoid vaccine coverage to be harmed by false alarms or by genuine adverse reactions approached inadequately or untimely.

\subsection{Vaccination and surveillance of adverse events in Brazil}

In Brazil, the vaccines included in the basic vaccination calendar are available for free in all primary health care units. The yellow fever vaccine is offered exclusively by the public service. Epidemiological surveillance system organized by the Ministry of Health collects and analyzes data on selected infectious diseases. SINAN (the acronym for the national system for notification of diseases) includes meningitis as one of the syndromes of mandatory reporting and investigation. Surveillance of adverse events following immunization (AEFI) has been conducted by the National Immunization Programme (NIP). The AEFI National Surveillance System processes data generated in a standardized form by vaccination teams and healthcare workers. 


\subsection{The yellow fever vaccine}

The yellow fever vaccine is generally considered safe. Common adverse events are mild and occur 5-7 days after vaccination. Revaccination is even safer with regard to events associated to viremia [3-6]. The vaccine virus' neurovirulence has been demonstrated in animal experiments and in encephalitis reports, mainly in children. The incidence of post-vaccination encephalitis was estimated as $0.5-4.0 / 1000$ in infants less than 6 months old [3,5], and as 1/1,000,000 or less in adults $[3,7]$. Post-vaccination encephalitis is characterized by the onset, 7-21 days after vaccination, of fever and variable neurological signs (including meningismus, convulsion, obtundation and paresis) associated to altered cerebral spinal fluid tests (100-500 cells and increased protein concentration) [3]. Clinical course is typically brief and recovery generally complete [3]. Although abnormal brain function is the important distinguishing feature between encephalitis and meningitis, this distinction is frequently blurred since some patients may have both a parenchymal and meningeal process with clinical features of both. Acknowledging the overlap, the term meningoencephalitis is frequently used for that condition.

Currently, yellow fever vaccine is given to children aged 9 months in endemic areas (Amazon region and parts of other six states). The efforts to avert the reintroduction of yellow fever in urban areas were intensified through yellow fever vaccination for all age groups. Of the 80 million doses applied since 1994, 34 million (43\%) were applied in 1999-2001 period. AEFI attributed to yellow fever vaccination rose from 0.06 to 1.32 per 100,000 vaccinees in Brazil, between 1998 and 2000 [4]. In that period three neurological events $(0.09$ per million) were reported (one encephalitis and two cases of paralysis) [4]. Since introduction of routine yellow fever vaccination in 1999, three yellow fever vaccination campaigns were held in Juiz de Fora, State of Minas Gerais, targeting different age groups: in 1999 children between 9 months and 5 years old; in 2000, people older than 60 years; and in 2001, the entire population. During the 2001 vaccination campaign held between March and April there was a rise in the number of reported neurological manifestations in Juiz de Fora characterised as aseptic meningitis temporally associated to yellow fever vaccination.
This article presents an analysis of notified AEFI cases following yellow fever vaccination held in a municipality where the resources and the challenges of the primary care network to deal with those issues are typical in Brazil. The study sought to expand the analysis of data from AEFI surveillance in the local level, particularly during mass vaccination campaigns. Of special interest is the analysis of aseptic meningitis for which the association with the yellow fever vaccine is still unclear.

\section{Materials and method}

The study analyzed all AEFI cases attributed to yellow fever vaccine notified in Juiz de Fora city, from January 1999 to December 2005. Juiz de Fora is a 457,000 inhabitants city located in the southeast region of Brazil. The sources of data for the study were (i) AEFI National Surveillance System notification forms; (ii) SINAN investigation forms for meningitis; (iii) records of administered doses of yellow fever vaccine. The case definition for aseptic meningitis considered as AEFI was based on (i) the time interval (up to 30 days) for the occurrence of neurological signs and symptoms following vaccination, (ii) clinical manifestation and abnormalities in cerebrospinal fluid consistent with aseptic meningitis, (iii) notification to the AEFI surveillance system, (iv) notification to the SINAN and (v) aseptic meningitis cases investigated and confirmed by the Epidemiology Department.

Data analysis was performed using the software SPSS Version 9.0. The denominator for estimation of notification rates was the number of doses of yellow fever vaccine in each study period. Neither denominator nor numerator data distinguished between primary and secondary vaccinees. The results were compared to data from the NIP and to published data.

\section{Results}

Of the 499,714 doses of yellow fever vaccine applied in Juiz de Fora between 1999 and 2005, 62.0\% (309,920 doses) were administered during the March-April 2001 vaccination campaign (90.0\% of the total of applied doses in 2001). In

Table 1

Annual rate (per 100,000 doses) of adverse events following immunization (AEFI) and aseptic meningitis rate (per 100,000 inhabitants)

\begin{tabular}{|c|c|c|c|c|c|}
\hline Year & $\operatorname{AEFI}(N)$ & Doses $(N)$ & Rate during campaign & Annual rate & Aseptic meningitis rate \\
\hline 1999 & 4 & 43,685 & 9.2 & 9.2 & 8.5 \\
\hline 2000 & 2 & 64,068 & 3.1 & 3.1 & 2.6 \\
\hline 2001 & 46 & 344,195 & 12.9 & 13.4 & 10.1 \\
\hline 2002 & 0 & 9,843 & - & - & 6.1 \\
\hline 2003 & 0 & 12,740 & - & - & 2.0 \\
\hline 2004 & 2 & 13,754 & - & 14.5 & 5.2 \\
\hline 2005 & 1 & 11,429 & - & 8.7 & 1.1 \\
\hline 1999-2005 & 55 & 499,714 & 11.0 & 11.0 & \\
\hline
\end{tabular}

Juiz de Fora, 1999-2005. 
Table 2

Rates of adverse event following immunization by age group

\begin{tabular}{lccc}
\hline Age group & AEFI $(\mathrm{N})$ & Doses $(\mathrm{N})$ & Rate per 100,000 \\
\hline$<$ 1 year old & 3 & 23,492 & 12.8 \\
1-5 years old & 3 & 51,841 & 5.8 \\
5-15 years old & 9 & 65,121 & 13.8 \\
15-59 years old & 33 & 352,490 & 9.4 \\
$>60$ years old & 3 & 46,739 & 6.4 \\
Not informed & 4 & - & -
\end{tabular}

Juiz de Fora, 1999-2005.

$1999,92.0 \%$ of doses were administered in children under 14 years of age (39,950 doses). In 2000, 62.5\% (40,024 doses) of vaccinees were over 60 years of age. In subsequent years, most doses were given in routine immunization of children.

The study analyzed 55 AEFI cases attributed to yellow fever vaccine during the 1999-2005 period. Most AEFI notifications attributed to yellow fever vaccine occurred in 2001 $(72.7 \%, n=40)$ and were concentrated in March and April. The AEFI notification rate reached a peak in 2001, when the annual notification rate was four times higher than the previous year (Table 1). Although most notified AEFI (60.0\%) occurred in the 15-59 years age group, the notification rate was similar in that age group (9.4 per 100,000 doses) and those younger than 5 years old $(8.0$ per 100,000$)$ (Table 2$)$. In $89.1 \%$ of all notifications the yellow fever vaccine had been the only vaccine administered. The vaccine had been applied during campaigns in $72.7 \%$ of the AEFI reported cases. The vaccines were within their expiration date and the AEFI involved different lot numbers.

There was no statistically significant difference in the distribution of cases by gender ( $49.1 \%$ were women). Systemic events accounted for $87.3 \%$ of notifications. Frequent clinical manifestations included fever $(n=44)$, vomiting $(n=39)$, headache $(n=21)$, meningismus $(n=9)$, myalgia $(n=10)$ and arthalgia $(n=8)$. Jaundice was described in two cases, including one with associated abdominal pain. Reports of neurological manifestation were limited to the year 2001 . Twenty-four (43.6\%) of systemic events received medical assistance in hospitals or emergency rooms.

An increment in the rate of aseptic meningitis (10.1 per 100,000 inhabitants; 3.87 per 100,000 doses) was observed in 2001 during the vaccination campaign period (Table 1 and Fig. 1). Fifty percent of the total notified as aseptic meningitis in SINAN in $2001(n=46)$ occurred in individuals older than 15 years old (Fig. 1). Of these, 12 were temporally linked to 2001 yellow fever vaccination (being 10 in April) and generated AEFI notification forms at the Epidemiology Department in Juiz de Fora. The median time between vaccination and meningitis was 17.5 days. The time interval for the beginning of fever episodes after yellow fever vaccination varied from 8 hours to 21 days, with a median of 5 days. All aseptic meningitis cases received medical care and were cured without sequelae. None of these notified cases presented fever or other symptoms within 72 hours before vaccination.

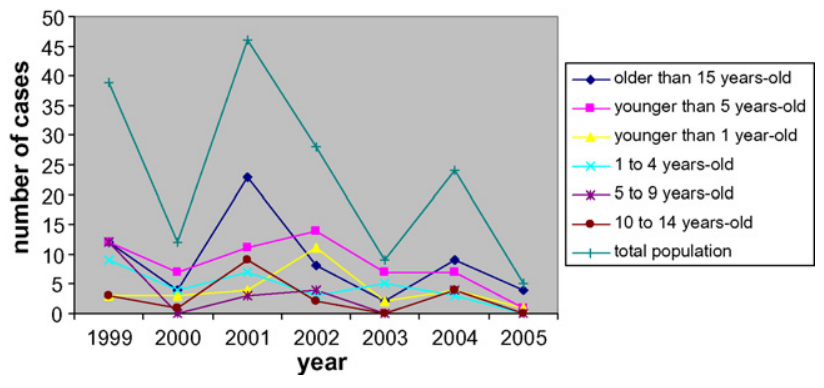

Fig. 1. Distribution by year and age group of aseptic meningitis cases, Juiz de Fora Brazil, 1999-2005.

\section{Discussion}

Limitations of passive surveillance systems (based on spontaneous reporting) of AEFI include (i) both undernotification and over-notification, (ii) problems inherent to completion of the notification form and data sources, (iii) poorly defined cases and (iv) inaccurate estimation of denominators. Partly filling those gaps a few observational epidemiological studies have investigated adverse events following immunization and estimated their frequency and association with vaccines [5,7-9,3].

In the first 7 years of yellow fever vaccination in Juiz de Fora, the most severe AEFI attributed to this vaccine was aseptic meningitis, and its clustering in time did not appear to have, in the reference period and region, other identifiable determinant factors. The vaccine coverage in the local population was $67.8 \%$ during the 2001 vaccination campaign and the cumulative coverage reached $98.8 \%$ in that year. With such a high proportion of the population exposed to the yellow fever vaccine, even a coincident outbreak of aseptic meningitis from other causes was likely to be attributed to the vaccine. The investigation of cases, along with available clinical, laboratory and epidemiological data, did not offer sufficient elements to assess the association with yellow fever vaccine. Firstly, many notified events were non-specific and could result from other health disturbances. Besides, vaccination against yellow fever had been recently introduced in the region and by that time, there were general concerns about the vaccine's safety, so that it could represent an easy and convenient explanation for cases with incomplete clinical and laboratory investigation. In the study scenario, the healthcare workers' perception of the existence of an association between yellow fever vaccine and neurological events may have misled both detection and notification of cases.

Temporal association between adverse events and vaccination is necessary, but insufficient to analyze causality [10]. The AEFI definition has low specificity [11] and data from passive notification may bias epidemiological studies [12]. In the present study, notification rates rather than incidence rates were estimated. Besides, the only available information was the occurrence of adverse events among vaccinees (outcome data among exposed individuals), thus hampering the estimation of relative risk $[13,14]$. 
The biological plausibility of that association is given by the vaccine virus neurovirulence, by previous reports of post-vaccination encephalitis and other neurologic manifestations and by the observed time between vaccination and symptoms [3]. However, there was not an effective clinical and laboratory investigation of suspected cases to support causality by the vaccine virus, and to make differential diagnosis.

Rates of notified AEFI varied substantially across age groups, but the difference may not be meaningful given that these rare events generated unstable rates (Table 2). The age groups affected by neurological events (older children and adults) were not in the risk age group for encephalitis reported in the literature: infants younger than 9 months and the elderly (no serious AEFI reported) [3,8,15]. The rate of notified AEFI against yellow fever observed in Juiz de Fora during the study period - 11.0/100,000 doses - was much higher than those estimated from NIP data between 1999 and $2001-0.75 / 100,000$ [16] and published data [3,17]. It was even higher (12.9 per 100,000 doses) during the 2001 vaccination campaign period, suggesting that surveillance could have been enhanced in that period. The 12 cases of aseptic meningitis notified in 2001 during the Juiz de Fora mass campaign indicate a notification rate of neurological events much higher $(3.87 / 100,000)$ than that observed in the rest of the study period $(0.0 / 100,000)$, for postvaccination encephalitis in Kenya in $1993(0.58 / 100,000)$ [3], in Ivory Coast during mass campaign vaccination in $2001(0.0 / 2,600,000)$ [7] and for neurotropic events in the United States, based on passive reporting data from 1990 to $2002(0.4 / 100,000)$ [15]. In the Brazilian NIP records there were four cases of aseptic meningitis among 13,715,643 doses applied in $2001(0.03 / 100,000)$ one encephalitis case in 2002 (4,444,393 doses) and no cases in 2003 (4,609,758 doses). In the city of Campinas, Brazil, there was an outbreak of aseptic meningitis temporally associated to the 2000 yellow fever vaccination campaign (unpublished data from Health Secretary from São Paulo). Seven hundred and twenty-three aseptic meningitis cases occurred during the vaccination period when 2,070,000 doses where applied (34.9 per 100,000). The time between vaccination and symptoms varied from 12 to 30 days. The investigation did not find clinical or laboratory evidence of association of cases to the vaccine.

The simultaneous increase in the utilization of yellow fever vaccines and implementation of the AEFI surveillance system may explain the increased detection of AEFI cases attributed to yellow fever vaccine in Brazil in the last few years. Vaccination campaigns and introduction of a new vaccination strategy may also influence the notification profiles. The higher incidence of notifications in mass campaigns may be due to an actual increase of AEFI incidence, to a change in perception of AEFIs or to coincidence of other diseases. In addition to the rate of AEFI, vaccination in mass campaign, which included all age groups, might have influenced the type of events attributed to the vaccine.
The investigation of possible AEFI outbreaks, even if supported or conducted by State or Federal officers, depends mainly on the ability to detect and analyze data locally [18]. Consolidated data for states and the whole country may be of limited use to identify AEFI related to local vaccination campaigns, since their effects are diluted in large data sets. Besides, information generated by analysis at a central level may not reach local level with the needed detail and swiftness to allow decision-making.

Identification of AEFI through notification of meningitis to SINAN enhanced the surveillance system's sensitivity. This finding associated to the notification of a vaccine related case of jaundice and abdominal pain in Juiz de Fora showed the preparedness for detecting AEFI from morbidity data (aseptic meningitis and viscerothropic disease).

The analysis presented indicated opportunities to improve data quality, mainly regarding the development of a standardized procedures protocol before implementation of a new vaccination strategy. In addition to the improvement of wellestablished routine AEFI surveillance, sentinel surveillance analogous to the protocol developed in Brazil for visceralization may provide clinical evidence strong in itself to support causality and to contribute to epidemiological studies. There is also room for improvement on aspects regarding data gathering and analysis: (i) baseline AEFI notification rates using data from routine vaccination; (ii) integrated analysis with infectious diseases surveillance data, AEFI passive surveillance system data and number of applied doses; (iii) local, regional and national data analysis, considering vaccination strategy and lot number used in different regions; (iv) clinical and laboratory investigation of suspected AEFI cases; (v) development of a standardized procedures protocol before implementation of a new vaccination strategy.

Yellow fever vaccine coverage has been suboptimal in Juiz de Fora city since 2001, because of safety concerns both from local population and from healthcare workers. The investigation has not preserved the public's confidence and has not avoided vaccine coverage to be harmed by false alarms or by genuine adverse reactions approached inadequately or untimely. The major challenge of AEFI surveillance systems is to address, based on epidemiological and clinical investigations, questions and hypotheses raised during daily activities of health services. The putative association of aseptic meningitis is a recurrent issue and should be targeted in future studies taking advantage of mass vaccination campaigns or using a case-control approach. Epidemiological studies should be routinely performed in order to: (i) assess public health interventions and health policies; (ii) help decisionmaking process; (iii) ensure public health interventions based on scientific criteria. Taking advantage of public health interventions (like mass vaccination campaigns or introduction of a new vaccination strategy), may provide larger datasets and the possibility to design epidemiological studies (e.g. case-control approach) and to link morbidity data and AEFI data. 


\section{References}

[1] Spier RE. Perception of risk of vaccine adverse events: a historical perspective. Vaccine 2002;20:S78-84.

[2] Fine PEM, Chen RT. Confounding in studies of adverse reactions to vaccines. Am J Epidemiol 1992;136(2):121-35.

[3] Monath TP. Yellow fever vaccine. In: Plotkin SA, Orenstein WA, editors. Vaccines. 4th ed. Philadelphia, WB: Saunders Company; 2004. p. 1095-176.

[4] Brasil Ministério da Saúde FUNASA. Eventos adversos sérios associados com a vacina 17D contra a febre amarela.

[5] CDC (Centers for Disease Control and Prevention). Notice to readers: fever, jaundice, and multiple organ system failure associated with 17Dderived yellow fever vaccination, 1996-2001. MMWR Morb Mortal Wkly Rep 2001;50(30):643-5.

[6] Camacho LAB, Aguiar SG, Freire MS, Leal ML, Nascimento JP, Igushi $\mathrm{T}$, et al. Reactogenicity of yellow fever vaccines in a randomised, placebo-controlled trial. Rev Saúde Públ 2005;39(3):413-20.

[7] Fitzner J, Coulibaly D, Kouadio DE, Yavo JC, Loukou YG, Koudou PO, et al. Safety of yellow fever vaccine during the September 2001 mass vaccination campaign in Abidjan, Ivory Coast. Vaccine 2004;23:156-62.

[8] Martin M, Weld LH, Tsai TF, Mootrey GT, Chen RT, Niu M, et al. Advanced age as risk factor for illness temporally associated with yellow fever vaccination. Emerg Infect Dis 2001;7(6):945-51.

[9] Struchiner CJ, Luz PM, Dourado I, Sato HK, Aguiar SG, Ribeiro JG, et al. Risk of fatal adverse events associated with 17DD yellow fever vaccine. Epidemiol Infect 2004;132:939-46.
[10] Rosenthal S, Chen RT. The reporting sensitivities of two passive surveillance systems for vaccine adverse events. Am J Public Health 1995;85(12):1706-9.

[11] Fine PEM. Methodological issues in the evaluation and monitoring of vaccine safety. Combined vaccines and simultaneous administration-current issues and perspectives. Ann NY Acad Sci 1995;759:300-8.

[12] Farrington CP, Nash J, Miller E. Case series analysis of adverse reactions to vaccines: a comparative evaluation. Am J Epidemiol 1996;143(11):1165-73.

[13] Chen RT, Orenstein WA. Epidemiologic methods in immunization programs. Epidemiol Rev 1996;18(2):99-117.

[14] Chen RT, Pool V, Takahashi H, Weniger BG, Patel B. Combination vaccines: postlicensure safety evaluation. Clin Infect Dis 2001;33(Suppl 4):S327-33.

[15] Khromava AY, Eidex RB, Weld LH, Kohl KS, Bradshaw RD, Chen RT, et al. Yellow fever vaccine: an update assessment of advanced age as a risk factor for serious adverse events. Vaccine 2005;23:325663.

[16] Brasil Ministério da Saúde. Fundação Nacional de Saúde CD-rom Encontro do PNI. Brasília, Centro Nacional de Epidemiologia/Coordenação Geral do PNI, 2003.

[17] CDC (Centers for Disease Control and Prevention). Adverse events associated with 17D-derived yellow fever vaccination-United States, 2001-2002. MMWR Morb Mortal Wkly Rep. 2002 51(44):989-93.

[18] Fernandes GC, Camacho LAB, Carvalho MS. Surveillance system of vaccine adverse events and local data analysis - the experience in a middle sized city in Brazil, 1999-2001. Vaccine 2005;23:2349-53. 